\title{
Toward forward-looking OCT needle tip vision of the spinal neuroforamen: animal studies
}

David T. Raphael, Changhuei Yang, Nancy Tresser, Jigang Wu, Yaoping Zhang, et al.

David T. Raphael, Changhuei Yang, Nancy Tresser, Jigang Wu, Yaoping Zhang, Felix I. Feldchtein, Linda Rever, "Toward forward-looking OCT needle tip vision of the spinal neuroforamen: animal studies," Proc. SPIE 6424, Photonic Therapeutics and Diagnostics III, 642429 (23 March 2007); doi: 10.1117/12.703188

SPIE. Event: SPIE BiOS, 2007, San Jose, California, United States 


\title{
TOWARD FORWARD-LOOKING OCT NEEDLE TIP VISION OF THE SPINAL NEUROFORAMEN: ANIMAL STUDIES
}

\author{
David T. Raphael ${ }^{\mathrm{a}}$, Changhuei Yang $^{\mathrm{b}}$, Nancy Tresser ${ }^{\mathrm{c}}$, Jigang $\mathrm{Wu}^{\mathrm{b}}$, Yaoping Zhang ${ }^{\mathrm{a}}$, \\ Felix Feldchtein ${ }^{c}$, Linda Rever ${ }^{\mathrm{a}}$ \\ ${ }^{a}$ Department of Anesthesiology, Keck School of Medicine, University of Southern California \\ Medical Center, Los Angeles, CA 90033; ${ }^{b}$ Department of Electrical Engineering, California Institute \\ of Technology, Pasadena, California 91125 ; ${ }^{\circ}$ Imalux Corporation, Cleveland, Ohio 44114
}

\begin{abstract}
Neurologic complications have been reported with spinal transforaminal injections. Causes include intraneural injection, plus embolization occlusion of the radicular artery with subsequent spinal cord infarction. ${ }^{1}$ Optical coherence tomography (OCT) is a non-invasive imaging modality, which is used to image tissue microstructure with very high resolution (less than 20 microns) in real-time. With a view toward needle tip OCT visualization of the spinal neuroforamen, we conducted animal studies to explore OCT imaging of paraspinal neurovascular structures. With institutional animal care committee approval, we performed ex-vivo and in situ OCT studies in a euthanized dog, pig, and rabbit. Image data was gathered on spinal nerve roots, dura, and brachial plexus. Two systems were used: frequency domain OCT imaging system developed at California Institute of Technology, and time domain Imalux NIRIS system with a $2.7 \mathrm{~mm}$ diameter probe. In a euthanized pig, excised dura was punctured with a 17-gauge Tuohy needle. FDOCT dural images of the puncture showed a subsurface cone-shaped defect. In a rabbit in situ study, puncture of the dura with a 26-gauge needle is imaged as a discontinuity. FDOCT imaging of both small artery and large arteries will be presented, along with H\&E and OCT images of the brachial plexus.
\end{abstract}

Keywords: optical coherence tomography, transforaminal injection, pain, dura, nerve, brachial plexus

\section{INTRODUCTION}

Cervical radicular pain, for which disc herniation and foraminal stenosis are the major causes, is believed to originate from nerve inflammation. This is the basis for injecting intervertebral steroids in conjunction with local anesthetics. The use of an intervertebral transforaminal route, as opposed to an interlaminar approach, allows the direct delivery of steroids and local anesthetics onto the target nerve. However, multiple major neurologic risks are associated with needle injection into the spinal neuroforamen, despite the use of fluoroscopic needle-positioning measures. An imaging technology is needed whereby needle tip vision is achieved of critical neurovascular and other structures at the injection site. With such a capability, neurologic risks would be minimized, and the risk of failure of intended therapeutic effects would be lessened. We first review the complications and current safety limitations of current intervertebral transforaminal injection techniques, and propose how these concerns might be addressed with optical coherence tomography (OCT).

Serious neurologic complications and deaths have been reported with cervical injection procedures, including severe anterior spinal artery syndrome with fatal spinal cord infarction ${ }^{2}$, bilateral cortical blindness owing to left vertebral artery puncture due to air/contrast dye injection ${ }^{3}$, quadriplegia ${ }^{4}$, complex regional pain syndrome secondary to nerve root needle trauma ${ }^{5}$, and fatal perforation of the left vertebral artery ${ }^{6}$. Many more cases are known to have occurred, but have not been reported in the literature for medico-legal reasons. Hence, the complication prevalence rate is unknown, but it is believed to be significantly higher than procedures performed in the lumbosacral area.

The likely cause of many such neurologic injuries is compromised perfusion in a radicular or medullary artery ${ }^{7}$. Particulate material in depot preparations of corticosteroids, or injected air, can serve as emboli that can be injected directly into an artery, or that can be alternatively pressurized through a vessel puncture site. The introduced emboli occlude the radicular or medullary artery and infarct the spinal cord.

Photonic Therapeutics and Diagnostics III, edited by Nikiforos Kollias, Bernard Choi, Haishan Zeng, Reza S. Malek, Brian J. Wong, Justus F. R. Ilgner, Kenton W. Gregory, Guillermo J. Tearney, Henry Hirschberg, Steen J. Madsen, Proc. of SPIE Vol. 6424, 642429, (2007) - 1605-7422/07/\$18 · doi: 10.1117/12.703188 
The limitations of safety measures used to minimize such complications are:

1.1 Limitations of Fluoroscopy

Fluoroscopy cannot image the spinal nerve, nor can it image the radicular and medullary arteries, except when there is accidental intra-arterial injection of contrast dye ${ }^{8}$.

1.2 Unreliable blood aspiration test.

In both cervical and lumbosacral ESIs, a positive flash or aspiration of blood is only predictive of intravascular injection in less than half of the documented intravascular responses. Therefore, aspiration before injection is not a consistently reliable means of preventing needle intravascular placement ${ }^{9} 110$.

1.3 Individual deviations from presumed working anatomy.

Practice guidelines for safe cervical transforaminal procedures urge operators to place the needle posteriorly in the intervertebral foramen dorsal to the spinal nerve in order to avoid any arteries, on the assumption that these vessels lie anterior to the spinal nerve ${ }^{11}{ }^{12}$. However, one study ${ }^{13}$ has demonstrated that radicular arteries can be seen posterior to the spinal nerves. Because the extent of such individual variation is unknown, operators cannot rely on the presumed working anatomy as an absolute guarantee of procedural safety.

1.4 Discontinuous imaging of target site.

A test injection of contrast medium is considered mandatory. But an operator may easily miss the fleeting image of a thin radicular artery branch upon injection. In such a case digital subtraction angiography might prove useful as a backup safety measure. However, an inadvertent intra-arterial injection might still occur after an ever so slight movement of the operator's hand, or even with a minimal displacement of the syringe needle or of the volume tubing subsequent to the digital angiogram.

1.5 Non-fusion of midline cervical and high thoracic ligamentum flavum.

For interlaminar midline approaches with spinal needle procedures, the loss-of-resistance technique relies upon a perceptible penetration of the ligamentum flavum. However, mid-line gaps in the ligamenta flava are frequent at cervical $(>50 \%)$ and high thoracic levels $(2-20 \%)$, but are rare at the T3 level and below. Hence, one cannot rely upon penetration of the ligamentum flavum as a perceptible barrier to interlaminar cervical placement of an epidural needle ${ }^{14}$.

\subsection{Optical coherence tomography (OCT)}

For the above reasons, we explored in a few animals the possible use of optical coherence tomography (OCT) as an imaging adjunct. OCT is the optical analog of B-mode ultrasound. It is a laser-based imaging modality that uses near infrared light to non-destructively image subsurface tissue structures, such as nerves, fat, muscle, blood vessels, and cartilage. The technique is based on comparing light reflected from objects to a reference signal to determine depth penetration (interferometry). With a Michelson interferometer, OCT measures the amount of the interference obtained from different points within the tissue by moving the mirror in the reference arm, which changes the distance light travels in that arm. Two and three-dimensional images are produced by scanning the beam across the sample and recording the reflection as a function of transverse position.

Optical coherence tomography ${ }^{1516171819}$ allows imaging through most tissues, including nerves and arteries, to a depth of up to $2 \mathrm{~mm}$ with good resolution ( 10 microns). Although this would appear to be a very limited viewing depth, it might be sufficient to allow identification of key neurovascular structures located immediately at the needle tip prior to injection. For pain interventionalists, a one or two millimeter change in location can make a significant difference complication-wise. It follows that, if OCT could image key structures near the needle tip, neural injury could be avoided by slightly withdrawing the needle.

We present data obtained from preliminary ex vivo and in situ animal studies (dog, pig, rabbit), with the purpose of identifying OCT image features of critical structures (nerve, vessel, dura) in front of a forward-looking OCT imaging system. The images were obtained with two different forward-imaging OCT systems: (1) a frequency-domain OCT imaging system developed at the California Institute of Technology, and (2) a time-domain Niris ${ }^{\mathrm{TM}}$ Imaging System (Imalux, Cleveland, Ohio) with the use of a portable handheld $2.7 \mathrm{~mm}$ diameter probe. 


\section{METHODOLOGY}

2.1 California Institute of Technology Swept Source Frequency-Domain Imaging System

The frequency-domain OCT (FDOCT) engine employed in this experiment is based on a swept laser source (Micron Optics si425-1300SL). The OCT light source, which had a central wavelength of $1310 \mathrm{~nm}$ with a bandwidth of $70 \mathrm{~nm}$, was coupled to a fiber-based Michelson interferometer. Axial line scanning rate was $500 \mathrm{~Hz}$. This system was used for ex vivo studies, with the dissected specimens placed in the imaging well of the system. The forward-looking OCT beam, in micrometer translational steps, scanned the fixed-position specimens in transverse and longitudinal views.

\subsection{Imalux Niris Time-Domain System (Figure 1)}

The Niris ${ }^{\mathrm{TM}}$ Imaging system from Imalux Corporation is a compact desktop, time-domain, OCT system that uses lowcoherent broadband light in the near infrared range. Its in-depth scanning range is $2.2 \mathrm{~mm}$ in air, and lateral scanning range is up to $2 \mathrm{~mm}$. Axial line scanning rate is $140 \mathrm{~Hz}$, resulting in image acquisition rate 1.5 seconds for 200x200 pixel frame. It employs a $2.7 \mathrm{~mm}$ outer diameter reusable, handheld forward-imaging probe, which was placed atop structures of interest to acquire the images for in situ studies.

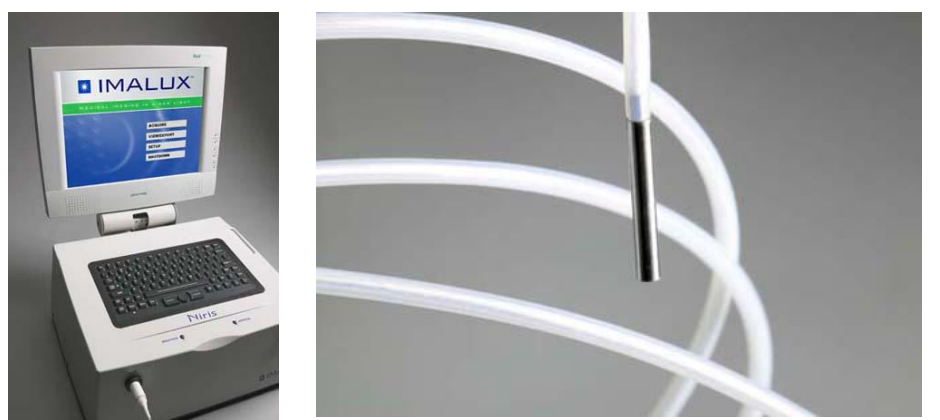

Figure 1. Imalux Niris OCT console (left) and reusable probe (right).

\subsection{Animal Studies}

All animal studies were performed with approval of the Animal Care Institutional Review Boards of the University of Southern California and the California Institute of Technology. All dissections were performed by a neurosurgeon, with careful exposure of paraspinal structures of interest. Epidural and spinal needles were used to create dural punctures, respectively, in a euthanized pig and rabbit. Dissected spinal nerve roots and radicular arteries in the intervertebral foramen region were studied in the same animals. The canine brachial plexus nerves were also studied. 


\section{DATA}
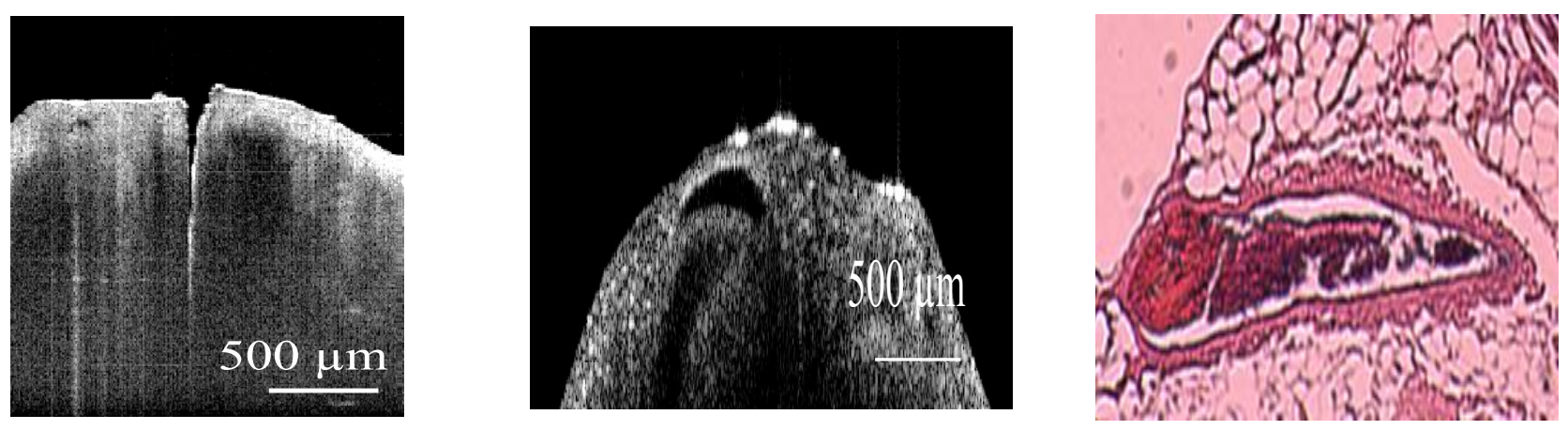

A

B

C

Figure 2. Ex Vivo Pig Study

In a $25 \mathrm{~kg}$ euthanized pig, surgical dissection was performed through the midline lumbar region down to the level of dura, along the pathway of an epidural needle midline-approach. A dural specimen was excised, and the ex vivo specimen was fixed in position, punctured with a 17-gauge Tuohy epidural needle, and placed in the imaging well of an FDOCT system A) Subsequent to puncture with the epidural needle, the effect on the dura is evident as a significant conical defect. Dissection was done to expose the lumbar spinal nerve roots in the neuroforamina of the euthanized pig. B) With the FDOCT system, we obtained ex-vivo images of a porcine radicular artery, which longitudinally followed the course of the spinal nerve root, and which was located on the posterior surface of the nerve. It has a muscularis layer, compatible with that of an artery. Of special note is the small size of the subsurface vessel $(\sim 500 \mu \mathrm{m})$. C) On the right hand side the corresponding hematoxylin-eosin stain reveals the same epineurial vessel, its half-moon shaped lumen partially filled with blood. Imaging was performed with the CalTech system.

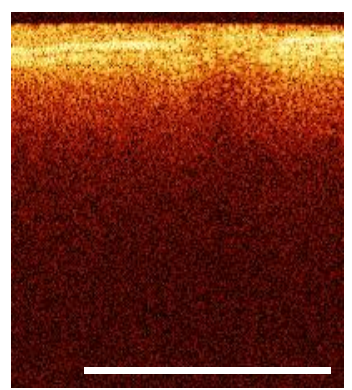

A

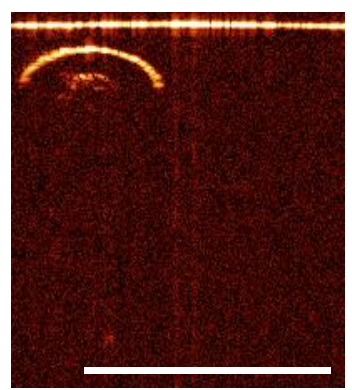

B

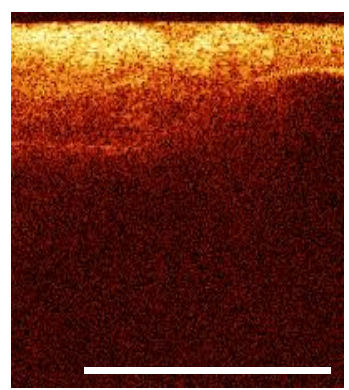

C

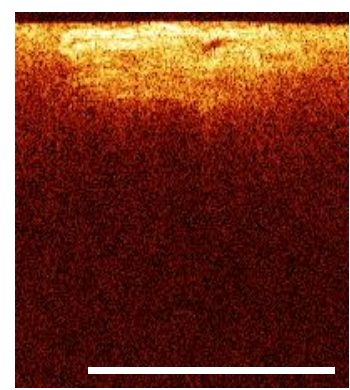

D

Figure 3 In Situ Rabbit Study

Dissection was performed on a euthanized $10 \mathrm{~kg}$ rabbit down to the lumbar dura, along the pathway of a spinal needle midline-approach. We performed successive dural punctures with a 26-gauge needle. A) Each puncture produced an evident discontinuity in the high scattering dural layer when visualized using OCT. The lateral size of the dural defect $(370 \mu \mathrm{m})$ was comparable to B) the needle tip $(400 \mu \mathrm{m})$. No similar defects were seen in the intact (unpunctured) dura. The dura in the rabbit measured $43 \mu \mathrm{m}$ in thickness. Dissection was done to expose the spinal nerve roots in a euthanized rabbit. No radicular vessel on a spinal nerve root was grossly seen during the rabbit dissection; however, additional OCT images revealed the presence of subsurface radicular vessels measuring approximately $260-300 \mu \mathrm{m}$ in diameter C) Spinal nerve roots. The OCT was able to visualize the lumbar spinal nerve roots, which were evident as bright homogeneous structures and D) the spinal artery (collapsed). OCT imaging was done with the time-domain Niris System. Bar is $1 \mathrm{~mm}$. 


\section{RESULTS}

With FDOCT imaging in a pig, the dural defect produced by the 17-g Tuohy needle has the shape of a cone. The defect is widest at the surface, and extends to an intradural depth of $1.2 \mathrm{~mm}$. The subsurface extension of the defect is not evident in conventional en face electron micrographs that show the defect frontally. It should be noted that the spinal dura mater is a tube of dense connective tissue that is generally not very vascular, except for tiny twigs from the spinal arteries. Hence, intradural vessels in these images are not seen, as expected.

With an in situ 26-gauge dural puncture in a rabbit, the defect appears as a discontinuity in the dura. The defect fills up with underlying cerebrospinal fluid. In comparison to the pig epidural defect, the spinal needle defect is not as evident in the depth direction.

The radicular artery is a critical feature that a pain interventionalist must recognize in order to avoid intravascular injection while advancing the needle in the transforaminal region. A porcine epineurial vessel is clearly imageable on top of a spinal nerve root in figures $2 \mathrm{~B}$ and $2 \mathrm{C}$. The evident muscularis layer distinguishes this structure as an artery, which is surrounded by fatty tissue, and which very likely is the posterior branch of a radicular artery. The crescent moon shape is compatible with the appearance of extrinsic nutrient arterial vessels that reach the epineurium, ramify within the outer layer, and supply the intraneural plexus through ascending and descending branches.

As shown in earlier studies ${ }^{16}$, OCT can be used to identify nerve fascicles, a characteristic and defining feature of peripheral nerves. An individual nerve fascicle, depending on its location and function, can be monofascicular, bifascicular, or polyfascicular. With a higher resolution OCT system, it may be possible to consistently image nerve fibers within the fascicles. Images of the canine brachial plexus nerve reveal a honeycomb of myelinated nerve fibers in transverse cross-section, as evident with hematoxylin-eosin staining. Tortuosity of the ex vivo fascicles within the nerve occurs because the elastic and normally stretched nerves retract upon excision ${ }^{17}$. With high-frequency ultrasound, the same tortuous pattern is seen in longitudinal images of ex-vivo nerve specimens ${ }^{20}$.

\section{CONCLUSIONS}

For pain specialists, the basic question is whether OCT imaging can prevent neural injury. Prior investigations indicate significant neural injury results when a needle penetrates through the fascicle, and even more so when local anesthetic is injected into the fascicle ${ }^{21}$. In contrast, little or no injury occurs when the needle path, even with local anesthetic injection or infusion, is extrafascicular, that is, into the epineurium ${ }^{21}{ }^{22}$. Certainly, if a nerve fascicle is seen at the tip of a forward-imaging OCT needle endoscope, it would make sense to withdraw the needle slightly.

What about injection into a vessel? For an artery, OCT can image the wall layers of the vessel, and allow its ready identification as such. Distinguishing an epineurial artery from a vein would require observing the thickness of the muscularis layer.

Hence, given the limited depth range of OCT, for an image of the tissue in the immediate vicinity of the needle tip, is the combined absence in the OCT image of a vessel and the absence of nerve fascicles a sufficiently protective measure against neural harm? The preliminary evidence presented herein allows us to think so. First, the conventional radiological guidance methodology could be employed to optimally position the needle tip. Second, OCT could be used to explore the proposed injection site, if need be, to determine whether a vessel or nerve was in the immediate vicinity. If a nerve or vessel was noted, the needle could be repositioned such that no neurovascular structure was present in the OCT image, indicating at least a $2 \mathrm{~mm}$ margin of safety, for which the risk of an intraneural or intravascular injection should be nil. On a cautionary side, we note that other imaging techniques have been proposed in which the absence of a single endpoint was deemed as safe, yet subsequent clinical usage revealed that it was found to be insufficient to provide complete safety or protection.

OCT has significant limitations. It is unlikely that it can be used as a major guidance method to direct the needle from skin to final foraminal injection site. For example, if the needle tip is $1-2 \mathrm{~cm}$ away from the target site, the currently available OCT in-depth penetration and scanning range of $2 \mathrm{~mm}$ may not reveal any identifiable landmark structures in the spinal foraminal area. Careful half-millimeter-sized movements of the needle would be needed to bring reference structures into view. The most suitable role of OCT would then seem to be to combine it with standard radiological 
positioning, and then to use it as an exclusionary high-resolution device for the final end positioning. At the very end, OCT could be used to exclude the possibility of any injection into a critical neurovascular structure. Such an approach might be superior to conventional blood aspiration and dye injection tests that have not proven to be sufficiently protective.

Present studies indicate that the effective imaging depth of OCT can be extended. Adaptive ranging (AR) is a dynamic feedback technique currently in development whereby image data are utilized to adjust the OCT system coherence gate offset, improve $\mathrm{dB}$ sensitivity, and extend the imaging range to as much as $7 \mathrm{~mm}^{23}$. If this extended imaging range is consistently attainable with AR-equipped OCT systems, OCT as an imaging modality would become much easier to use; however, the tradeoff associated with compromised lateral resolution and reduced image brightness consistent with larger focal depth should be carefully evaluated.

The major remaining limitation is thinness of the imaging probe. Current research is proceeding toward the creation of even thinner OCT needle probes. Ideally, it would seem that practitioners need a 25 -g forward-looking OCT needle for cervical foraminal injections. However, the smallest forward-looking OCT imaging needle currently in existence is a frequency-domain 21-gauge imaging needle developed at CalTech ${ }^{24}$. This is close in size to a 22-gauge spinal needle, which is used often in lumbosacral spinal injection procedures. Any such OCT needle probe, with its internal optical fiber incorporated into the needle, would also require a conduit for the administration of injectate. In its proposed design, the OCT needle endoscope would allow needle tip visualization of the actual site of injection and of the local spread of the injectate.

These preliminary animal experiments suggest a potential niche for an OCT-based imaging needle in the performance of spinal foraminal injections. However, many more investigations involving humans are required before OCT can be recommended for routine use. An extensive OCT neuroforaminal image database first needs to be created, in order to better appreciate the effects of individual variation and disease processes.

\section{REFERENCES}

1. M. B. Furman, M. T. Giovanniello, E. M. O'brien, "Incidence of intravascular penetration in transforaminal cervical epidural steroid injections," Spine, 28, 1, 21-25, 2003.

2. P. J. Brouwers, E. J. Kottink, M. A. Simon, et al., "A cervical anterior spinal artery syndrome after diagnostic blockade of the right C6-nerve root," Pain, 91, 3, 397-399, 2001.

3. M. R. Mcmillan, C. Crumpton, "Cortical blindness and neurologic injury complicating cervical transforaminal injection for cervical radiculopathy," Anesthesiology, 99, 2, 509-511, 2003.

4. $\quad$ Anonymous, "Miss-'n-mix and mimics. Tetraplegia following erroneous epidural injection. ," Acta Anaesthesiol Belg., 52, 205-206, 2001.

5. K. Wiesauera, M. Pircherb, E. Gutzingerb, et al., "En-face scanning optical coherence tomography with ultrahigh resolution for material investigation," Optics Express, 13, 3, 1015-1024, 2005.

6. L. Rozin, R. Rozin, S. A. Koehler, et al., "Death during transforaminal epidural steroid nerve root block (C7) due to perforation of the left vertebral artery," Am J Forensic Med Pathol, 24, 4, 351-355, 2003.

7. R. Baker, P. Dreyfuss, S. Mercer, et al., "Cervical transforaminal injection of corticosteroids into a radicular artery: a possible mechanism for spinal cord injury," Pain, 103, 1-2, 211-215, 2003.

8. J. P. Rathmell, C. Aprill, N. Bogduk, "Cervical transforaminal injection of steroids," Anesthesiology, 100, 6, 1595-1600, 2004.

9. M. B. Furman, E. M. O'brien, T. M. Zgleszewski, "Incidence of intravascular penetration in transforaminal lumbosacral epidural steroid injections," Spine, 25, 20, 2628-2632, 2000.

10. J. K. Houten, T. J. Errico, "Paraplegia after lumbosacral nerve root block: report of three cases," Spine J, 2, 1, 70-75, 2002.

11. B. G. Chakravorty, "Arterial supply of the cervical spinal cord (with special reference to the radicular arteries)," Anat Rec, 170, 3, 311-329, 1971.

12. I. M. Turnbull, A. Brieg, O. Hassler, "Blood supply of cervical spinal cord in man. A microangiographic cadaver study," J Neurosurg, 24, 6, 951-965, 1966.

13. H. Crock, An Atlas of Vascular Anatomy of the Skeleton and Spinal Cord, Pages, Martin Duntz, London, 1996. 
14. P. Lirk, C. Kolbitsch, G. Putz, et al., "Cervical and high thoracic ligamentum flavum frequently fails to fuse in the midline," Anesthesiology, 99, 6, 1387-1390, 2003.

15. D. Huang, E. A. Swanson, C. P. Lin, et al., "Optical Coherence Tomography," Science, 254, 1178-1181, 1991.

16. S. A. Boppart, B. E. Bouma, C. Pitris, et al., "Intraoperative assessment of microsurgery with three-dimensional optical coherence tomography," Radiology, 208, 1, 81-86, 1998.

17. D. T. Raphael, J. Zhang, Y. Zhang, et al., "OCT/PS-OCT Imaging of Brachial Plexus Neurovascular Structures," 5312, 388-395, City, 2004.

18. Z. H. Ding, Y. H. Zhao, H. W. Ren, et al., "Real-time phase-resolved optical coherence tomography and optical Doppler tomography," Optics Express, 10, 5, 236-245, 2002.

19. M. A. Choma, M. V. Sarunic, C. H. Yang, et al., "Sensitivity advantage of swept source and Fourier domain optical coherence tomography," Optics Express, 11, 18, 2183-2189, 2003.

20. W. T. Yang, P. T. Chui, C. Metreweli, "Anatomy of the normal brachial plexus revealed by sonography and the role of sonographic guidance in anesthesia of the brachial plexus," AJR Am J Roentgenol, 171, 6, 1631-1636, 1998.

21. F. Gentili, A. R. Hudson, D. Hunter, et al., "Nerve injection injury with local anesthetic agents: a light and electron microscopic, fluorescent microscopic, and horseradish peroxidase study," Neurosurgery, 6, 3, 263-272, 1980.

22. J. K. Terzis, K. L. Smith, The Peripheral Nerve: Structure, Function and Reconstruction, Pages, Raven Press, New York, 1990.

23. N. V. Iftimia, B. E. Bouma, J. F. De Boer, et al., "Adaptive ranging for optical coherence tomography," Optics Express, 12, 17, 4025-4034, 2004.

24. J. Wu, M. Conry, C. Gu, et al., "Paired-angle-rotation scanning optical coherence tomography forward-imaging probe," Opt Lett, 31, 9, 1265-1267, 2006. 